\title{
IMPROVED LOWER BOUNDS FOR THE MOTION OF MOVING BOUNDARIES
}

\author{
JAMES M. HILL and JEFFREY N. DEWYNNE ${ }^{1}$
}

(Received 13 October 1983)

\begin{abstract}
In a recent paper the authors give upper and lower bounds for the motion of the moving boundary for the classical Stefan problem for plane, cylindrical and spherical geometries. On comparison with the exact Neumann solution for the plane geometry and no surface radiation the bounds obtained are seen to be quite adequate for practical purposes except for the lower bound at small Stefan numbers. Here improved lower bounds are obtained which in some measure remove this inadequacy. Time dependent surface conditions are also examined and the new lower bounds obtained for the classical problem are illustrated numerically,
\end{abstract}

\section{Introduction}

A number of important problems in metallurgy and chemical engineering involve unknown moving boundaries. In a recent paper [2] we have shown that the motion of the moving boundary for problems arising in the freezing or chemical reaction of spheres, cylinders and slabs can be bounded above and below. These results are achieved by utilizing an integral formulation of the problem and establishing the pseudo steady state solution for the temperature (concentration) as an upper bound to the actual temperature (concentration). For large Stefan number $\alpha$, the bounds obtained in [2] are adequate as simple engineering approximations. However in the steel industry, for example, the relevant value of the Stefan number is approximately 0.25 and therefore it is desirable to obtain more accurate bounds for small Stefan numbers. Further if we compare the numerical results from [2] for the case for which the exact Neumann solution is available then it becomes apparent that the lower bound is a considerably less

\footnotetext{
${ }^{1}$ Department of Mathematics, The University of Wollongong, Wollongong, N.S.W. 2500

(c) Copyright Australian Mathematical Society 1984, Serial-fee code 0334-2700/84
} 
tight bound on the actual solution than is the upper bound. The purpose of this paper is to extend the results in [2] by improving the lower bound for the motion of the moving boundary.

In nondimensional variables the classical Stefan problem with Newtonian cooling on the surface of an infinite half-space $(-\infty, 1)$, an infinite circular cylinder or a sphere can be formulated as follows:

$$
\begin{gathered}
\frac{\partial c}{\partial t}=\frac{\partial^{2} c}{\partial r^{2}}+\frac{\lambda}{r} \frac{\partial c}{\partial r}, \quad R(t)<r<1, \\
c(1, t)+\beta \frac{\partial c}{\partial r}(1, t)=1, \quad c(R(t), t)=0, \\
\frac{\partial c}{\partial r}(R(t), t)=-\alpha \dot{R}(t), \quad R(0)=1,
\end{gathered}
$$

where $c(r, t)$ and $R(t)$ denote the nondimensional temperature (concentration) and the position of the moving boundary at time $t$ respectively. The constants $\alpha$ and $\beta$ are respectively positive and non-negative, and $\lambda$ is 0,1 or 2 for the half-space, cylinder and sphere respectively. It is important to note that the problem is nondimensionalised such that $0 \leqslant c(r, t) \leqslant 1$. The only exact solution for the above problem is the classical Neumann solution for $\beta$ and $\lambda$ zero, which is given by

$$
c(r, t)=1-\frac{\operatorname{erf}\left[(1-r) / 2 t^{1 / 2}\right]}{\operatorname{erf}\left[(\gamma / 2)^{1 / 2}\right]}, \quad R(t)=1-(2 \gamma t)^{1 / 2},
$$

where $\gamma$ satisfies the transcendental equation

$$
\alpha(\pi \gamma / 2)^{1 / 2} \exp (\gamma / 2) \operatorname{erf}\left[(\gamma / 2)^{1 / 2}\right]=1 .
$$

Of particular practical interest is the time $t_{\mathrm{c}}$ for the moving boundary to reach the origin $\left(R\left(t_{\mathrm{c}}\right)=0\right)$ which we refer to as the time to complete reaction. For the half-space this definition is of course artificial but is nevertheless useful for comparison purposes.

In the following section we summarise the main results of [2], but utilising the function $K_{\lambda}(x, y)$ defined by (2.1) which avoids repeating similar formulae for the three geometries considered. As far as the authors are aware this function has not been employed previously. In Section 3 we outline the procedure for the determination of the improved lower bounds to the motion of the boundary. In the section thereafter we summarise the results obtained for the new lower bounds for the three geometries. In Section 5 we describe how the procedure may be adapted to obtain bounds for the classical Stefan problem with a time dependent surface condition of the form (5.1). Numerical results are given in the final section. Finally in this section we refer the reader to Davis and Hill [1] for 
references specifically relating to the above problem. For more general moving boundary problem references the reader may consult Rubinstein [6], Ockendon and Hodgkins [5] and Wilson, Solomon and Boggs [7].

\section{Summary of known results}

The results obtained in [2] can be summarised most compactly in terms of the function

$$
K_{\lambda}(x, y)=\int_{y}^{x} \xi^{-\lambda} d \xi
$$

As described in [2] we find that $c(r, t)$ satisfies the integral equation

$$
c(r, t)=\frac{\partial}{\partial t} \int_{R(t)}^{r} \xi^{\lambda} K_{\lambda}(r, \xi)[\alpha+c(\xi, t)] d \xi,
$$

while the motion of the moving boundary is determined from

$$
t=\int_{R(t)}^{1} \xi^{\lambda}\left[\beta+K_{\lambda}(1, \xi)\right][\alpha+c(\xi, t)] d \xi .
$$

We remark that from (2.3) and the inequalities $0 \leqslant c(r, t) \leqslant 1$ we have immediately

$$
\alpha \Sigma_{\lambda}(R) \leqslant t \leqslant(\alpha+1) \Sigma_{\lambda}(R),
$$

where the function $\Sigma_{\lambda}(R)$ is defined by

$$
\Sigma_{\lambda}(R)=\int_{R}^{1} \xi^{\lambda}\left[\beta+K_{\lambda}(1, \xi)\right] d \xi .
$$

In the above terminology the standard pseudo steady state approximation to (1.1)-(1.3) can be shown to be given by

$$
c_{\mathrm{pss}}(r, R)=\frac{K_{\lambda}(r, R)}{\left[\beta+K_{\lambda}(1, R)\right]}, \quad t_{\mathrm{pss}}(R)=\alpha \Sigma_{\lambda}(R) .
$$

We remark that strictly speaking in (2.6) we should distinguish the pseudo steady state motion $R_{\mathrm{pss}}(t)$ defined by (2.6b). However in the following we need to refer to the pseudo steady state concentration and motion as functions of the actual motion $R(t)$, and therefore we dispense with the distinction. In [2] we show that

$$
0 \leqslant c(r, t) \leqslant c_{\mathrm{pss}}(r, R),
$$

so that from (2.3) and (2.7) we obtain

$$
\alpha \Sigma_{\lambda}(R) \leqslant t \leqslant \alpha \Sigma_{\lambda}(R)+\left[\beta+K_{\lambda}(1, R)\right]^{-1} \int_{R}^{1} \xi^{\lambda}\left[\beta+K_{\lambda}(1, \xi)\right] K_{\lambda}(\xi, R) d \xi .
$$


It is interesting to note that for the three geometries considered the upper bound in (2.8) improves that of (2.4). However for the cylinder and the sphere the upper bounds from (2.8) and (2.4) for the time to complete reaction actually coincide. This leads the authors to suspect that the upper bounds for $t_{c}$ obtained in [2] are probably as good as can be achieved from simple considerations. From (2.4) we find that

$$
\frac{\alpha(1+2 \beta)}{2(\lambda+1)} \leqslant t_{\mathrm{c}} \leqslant \frac{(\alpha+1)(1+2 \beta)}{2(\lambda+1)},
$$

and (2.8) yields the same result for cylinders and spheres while for the infinite half-space we obtain

$$
\frac{\alpha(1+2 \beta)}{2} \leqslant t_{\mathrm{c}} \leqslant \frac{\alpha(1+2 \beta)}{2}+\frac{(1+3 \beta)}{6(1+\beta)},
$$

and the upper bound in (2.10) is an improvement on that in (2.9) for $\lambda=0$. From the exact solution (1.4) for $\beta=0$ and (2.10) we have

$$
\alpha \leqslant \gamma^{-1} \leqslant \alpha+\frac{1}{3} \text {, }
$$

where $\gamma$ is a root of (1.5). These inequalities can be proved analytically and inspection of Table 1 verifies them numerically. From Table 1 it is also apparent that for small values of $\alpha$ the lower bound is considerably less tight a bound than is the upper bound.

\begin{tabular}{|c|c|c|c|c|}
\hline \multicolumn{3}{|c|}{$\begin{array}{c}\text { Lower } \\
\text { Bounds }\end{array}$} & $\begin{array}{c}\text { Exact } \\
\text { Solution }\end{array}$ & $\begin{array}{c}\text { Upper } \\
\text { Bound }\end{array}$ \\
\hline$\alpha$ & $(6.1 \mathrm{a})$ & $(6.1 \mathrm{~b})$ & $\gamma^{-1}$ & $\alpha+\frac{1}{3}$ \\
\hline 0.20 & 0.33 & 0.33 & 0.45 & 0.53 \\
\hline 0.25 & 0.38 & 0.38 & 0.50 & 0.58 \\
\hline 0.50 & 0.65 & 0.62 & 0.78 & 0.83 \\
\hline 1.00 & 1.15 & 1.12 & 1.30 & 1.33 \\
\hline 2.00 & 2.16 & 2.12 & 2.31 & 2.33 \\
\hline 5.00 & 5.16 & 5.11 & 5.33 & 5.33 \\
\hline 10.00 & 10.17 & 10.11 & 10.33 & 10.33 \\
\hline 50.00 & 50.17 & 50.11 & 50.33 & 50.33 \\
\hline 100.00 & 100.17 & 100.11 & 100.33 & 100.33 \\
\hline 500.00 & 500.17 & 500.11 & 500.33 & 500.33 \\
\hline
\end{tabular}

TABLE 1. Demonstration of the validity and utility of bounds with exact solution for $\lambda=0$ and $\beta=0$. 
In attempting to improve the lower bound for the motion the most obvious approach, at first sight, is to use the inequality

$$
-\alpha \dot{R}(t) R^{\lambda} K_{\lambda}(r, R) \leqslant c(r, t),
$$

which follows immediately from (2.2) on performing the time differentiation and noting that $\partial c / \partial t$ is positive. This approach involves at some stage obtaining a lower bound for the velocity $-\dot{R}(t)$ which appears to be a nontrivial problem. The procedure adopted here is essentially one of the techniques employed by Glasser and Kern [3] for half-space problems, although because of different notations and terminology this correspondence is not immediately apparent. We also remark that an alternative proof of the right-hand inequality in (2.7) is given in [4] using Green's functions for the spatial operator in the diffusion equation.

\section{Improved lower bounds}

In this section we describe in general terms the procedure for obtaining improved lower bounds while in the following section we detail specific results for the three geometries considered. We need the inequality

$$
-\frac{1}{\dot{R}(t)} \geqslant \alpha R^{\lambda}\left[\beta+K_{\lambda}(1, R)\right]=-\frac{d t_{\mathrm{pss}}}{d R}(R),
$$

which follows from (2.5) and (2.6b) and differentiating (2.3) with respect to $t$ and again noting that $\partial c / \partial t \geqslant 0$. From (2.2) and (2.3) we have

$t=t_{\mathrm{pss}}(R)+\frac{d}{d t} \int_{R}^{1} \int_{R}^{\xi}(\xi \eta)^{\lambda}\left[\beta+K_{\lambda}(1, \xi)\right] K_{\lambda}(\xi, \eta)[\alpha+c(\eta, t)] d \eta d \xi$,

which, upon integration yields

$$
\begin{aligned}
\frac{t^{2}}{2}= & \int_{0}^{t} t_{\mathrm{pss}}[R(\tau)] d \tau \\
& +\int_{R}^{1} \int_{R}^{\xi}(\xi \eta)^{\lambda}\left[\beta+K_{\lambda}(1, \xi)\right] K_{\lambda}(\xi, \eta)[\alpha+c(\eta, t)] d \eta d \xi .
\end{aligned}
$$

For the first integral in (3.3) we have using (3.1)

$$
\int_{0}^{t} t_{\mathrm{pss}}[R(\tau)] d \tau=\int_{R}^{1} \frac{-t_{\mathrm{pss}}(R)}{\dot{R}(\tau)} d R \geqslant \frac{t_{\mathrm{pss}}^{2}(R)}{2},
$$

while for the double integral in (3.3) we may use $c(r, t) \geqslant 0$ so that altogether we obtain

$$
t^{2} \geqslant t_{\mathrm{pss}}^{2}(R)+2 \alpha \int_{R}^{1} \int_{R}^{\xi}(\xi \eta)^{\lambda}\left[\beta+K_{\lambda}(1, \xi)\right] K_{\lambda}(\xi, \eta) d \eta d \xi .
$$


Since the integrand is a positive function it is apparent that (3.5) is an improvement on the lower bound given in the previous section.

In principle we may continue this process. However successive bounds, although always providing an improvement on $t_{\mathrm{pss}}(R)$ do not always generate tighter bounds than the previous one. On utilising (2.2) a further integration of (3.3) yields

$$
\begin{aligned}
\frac{t^{3}}{6} & =\int_{0}^{t} \int_{0}^{\tau} t_{\mathrm{pss}}[R(\nu)] d \nu d \tau \\
& +\alpha \int_{0}^{t} \int_{R(\tau)}^{1} \int_{R(\tau)}^{\xi}(\xi \eta)^{\lambda}\left[\beta+K_{\lambda}(1, \xi)\right] K_{\lambda}(\xi, \eta) d \eta d \xi d \tau \\
& +\int_{R}^{1} \int_{R}^{\xi} \int_{R}^{\eta}(\xi \eta \zeta)^{\lambda}\left[\beta+K_{\lambda}(1, \xi)\right] K_{\lambda}(\xi, \eta) K_{\lambda}(\eta, \zeta)[\alpha+c(\zeta, t)] d \zeta d \eta d \xi,
\end{aligned}
$$

which on using (3.1) and $c(r, t) \geqslant 0$ gives

$$
\begin{aligned}
t^{3} \geqslant & t_{\mathrm{pss}}^{3}(R) \\
& +6 \alpha^{2} \int_{R}^{1} \int_{\zeta}^{1} \int_{\zeta}^{\xi}(\xi \eta \zeta)^{\lambda}\left[\beta+K_{\lambda}(1, \xi)\right] K_{\lambda}(\xi, \eta)\left[\beta+K_{\lambda}(1, \zeta)\right] d \eta d \xi d \zeta \\
& +6 \alpha \int_{R}^{1} \int_{R}^{\xi} \int_{R}^{\eta}(\xi \eta \zeta)^{\lambda}\left[\beta+K_{\lambda}(1, \xi)\right] K_{\lambda}(\xi, \eta) K_{\lambda}(\eta, \zeta) d \zeta d \eta d \xi
\end{aligned}
$$

From numerical values for the bounds for $t_{\mathrm{c}}$ obtained from (3.5) and (3.7) with $R=0$ given in the final section, it is apparent for all but the smallest values of $\alpha$ and $\beta$ that (3.7) gives an inferior result to that obtained from (3.5). We might expect this phenomenon since the amount 'given away' by inequality (3.1) would eventually exceed the improvements obtained by substituting (2.2) at each step.

\section{Formulae for various geometries}

From (2.1), (2.2) and (2.6b) we find that (3.5) yields the following improved lower bounds for the motion of the moving boundary. For plane, cylindrical and spherical geometries we obtain respectively,

$$
\begin{aligned}
& t^{2} \geqslant \frac{\alpha^{2}}{4}(1-R)^{2}(1+2 \beta-R)^{2}+\frac{\alpha}{12}(1-R)^{3}(1+4 \beta-R) \quad(\lambda=0), \\
& t^{2} \geqslant \frac{\alpha^{2}}{16}\left[(1+2 \beta)\left(1-R^{2}\right)+2 R^{2} \log R\right]^{2} \\
&+\frac{\alpha}{32}\left[\left(1-R^{2}\right)\left(1+4 \beta+(5+4 \beta) R^{2}\right)+4 R^{2}\left(2+4 \beta+R^{2}\right) \log R\right] \\
&(\lambda=1),
\end{aligned}
$$




$$
\begin{aligned}
t^{2} \geqslant & \frac{\alpha^{2}}{36}(1-R)^{2}\left[(1+2 \beta)(1+R)+2(\beta-1) R^{2}\right]^{2} \\
& +\frac{\alpha}{60}(1-R)^{3}\left[(1-R)(1+4 R)+4 \beta\left(1+3 R+R^{2}\right)\right] \quad(\lambda=2) .
\end{aligned}
$$

Similarly (3.7) simplifies to yield,

$$
\begin{gathered}
t^{3} \geqslant \frac{\alpha^{3}}{8}(1-R)^{3}(1+2 \beta-R)^{3} \\
+\frac{\alpha^{2}}{24}(1-R)^{4}\left[(1-R)^{2}+6 \beta(1-R)+6 \beta^{2}\right] \\
+\frac{\alpha}{120}(1-R)^{5}(1+6 \beta-R) \quad(\lambda=0), \\
t^{3} \geqslant \frac{\alpha^{3}}{64}\left[(1+2 \beta)\left(1-R^{2}\right)+2 R^{2} \log R\right]^{3} \\
+\frac{\alpha^{2}}{1152}\left[( R ^ { 2 } - 1 ) \left(\left(72 \beta^{2}+114 \beta+19\right) R^{4}\right.\right. \\
\left.+\left(180 \beta^{2}+114 \beta+19\right) R^{2}-4\left(9 \beta^{2}+12 \beta+2\right)\right) \\
+6\left((19+24 \beta) R^{4}+72 \beta(1+\beta) R^{2}-9(1+4 \beta)\right) R^{2} \log R \\
+\frac{\alpha}{384}\left[\left(1-R^{2}\right)\left(10 R^{4}+19 R^{2}+1\right)+6\left(R^{4}+6 R^{2}+3\right) R^{2} \log R\right. \\
\left.+6 \beta\left(\left(1-R^{2}\right)\left(R^{4}+10 R^{2}+1\right)+12\left(R^{2}+1\right) R^{2} \log R\right)\right] \\
+\frac{\alpha}{840}(1-R)^{5}\left[(1-R)(1+6 R)+6 \beta\left(1+5 R+R^{2}\right)\right] \quad(\lambda=2) \\
+\frac{\alpha^{2}}{840}(1-R)^{4}\left[(1-R)^{2}\left(21 R^{2}+12+2\right)\right. \\
t^{3} \geqslant \frac{\alpha^{3}}{216}(1-R)^{3}\left[(1+2 \beta)(1+R)\left(7 R^{4}+12 R^{3}+10 R^{2}+4 R+1\right)\right]
\end{gathered}
$$


For the time to complete reaction $t_{\mathrm{c}}$ we find from (4.1), (4.2) and (4.3), with $R$ zero, that the first improved lower bound for the three geometries can be summarised by the following formula,

$$
t_{\mathrm{c}}^{2} \geqslant\left(\frac{\alpha(1+2 \beta)}{2(\lambda+1)}\right)^{2}+\frac{\alpha(1+4 \beta)}{4(\lambda+1)(\lambda+3)} .
$$

The general formula for the improved lower bound for $t_{\mathrm{c}}$ resulting from (4.4), (4.5) and (4.6) is not as apparent as (4.7). However from (2.1) and (3.7), with arbitrary $\lambda$, we may show directly, after a long calculation, that

$$
\begin{aligned}
t_{\mathrm{c}}^{3} \geqslant & \left(\frac{\alpha(1+2 \beta)}{2(\lambda+1)}\right)^{3} \\
& +\frac{\alpha^{2}\left(\lambda^{2}+5 \lambda+10\right)}{8(\lambda+1)^{2}(\lambda+2)(\lambda+3)(\lambda+5)}\left\{1+6 \beta+\frac{12 \beta^{2}(\lambda+5)}{\left(\lambda^{2}+5 \lambda+10\right)}\right\} \\
& +\frac{\alpha(1+6 \beta)}{8(\lambda+1)(\lambda+3)(\lambda+5)},
\end{aligned}
$$

and this result is in agreement with those obtained from (4.4), (4.5) and (4.6), with $R$ zero.

\section{Time dependent surface conditions}

In this section we consider the problem (1.1), (1.2) and (1.3) with (1.2a) replaced by

$$
c(1, t)+\beta \frac{\partial c}{\partial r}(1, t)=f(t)
$$

where $f(t)$ is assumed to be a positive monotonically increasing function of time. For this problem (2.2) still holds, but (2.3) is replaced by

$$
g(t)=\int_{R(t)}^{1} \xi^{\lambda}\left[\beta+K_{\lambda}(1, \xi)\right][\alpha+c(\xi, t)] d \xi
$$

where $g(t)$ is defined by

$$
g(t)=\int_{0}^{t} f(\tau) d \tau
$$

Upper and lower bounds for the motion of the boundary obtained from (2.7) and (5.2) are given in [2] for the sphere. In the general notation of this paper $g(t)$ and $g\left(t_{\mathrm{c}}\right)$ are bounded above and below by precisely the upper and lower bounds in (2.8) and (2.9) respectively. 
In order to deduce an improved lower bound for the motion of the boundary, we have from the time derivative of (5.2) and the assumed monotonicity of $f(t)$

$$
-\alpha \dot{R}(t) R^{\lambda}\left[\beta+K_{\lambda}(1, R)\right] \leqslant f(t) \leqslant f\left(t_{\mathrm{c}}\right),
$$

and therefore that

$$
-\frac{1}{\dot{R}(t)} \geqslant \frac{\alpha R^{\lambda}}{f\left(t_{\mathrm{c}}\right)}\left[\beta+K_{\lambda}(1, R)\right] .
$$

Thus proceeding as in Section 3 we have in place of (3.5)

$$
\int_{0}^{t} g(\tau) d \tau \geqslant \frac{\alpha^{2}}{2 f\left(t_{\mathrm{c}}\right)} \Sigma_{\lambda}(R)^{2}+\alpha \int_{R}^{1} \int_{R}^{\xi}(\xi \eta)^{\lambda}\left[\beta+K_{\lambda}(1, \xi)\right] K_{\lambda}(\xi, \eta) d \eta d \xi
$$

This equation represents the appropriate generalization for obtaining improved lower bounds with a time dependent surface condition. However the problem of whether (5.6) represents an improvement on $g(t) \geqslant \alpha \Sigma_{\lambda}(R)$ will in general depend on $f(t), \alpha$ and $\beta$.

For example for $f(t)=t$ we have from (2.9) that the pseudo steady state lower bound for $t_{\mathrm{c}}$ is given by

$$
t_{\mathrm{pssc}}=\left(\frac{\alpha(1+2 \beta)}{(\lambda+1)}\right)^{1 / 2}
$$

However from (5.6) we have

$$
4 t_{\mathrm{c}}^{4}-\frac{3 \alpha(1+4 \beta)}{(\lambda+1)(\lambda+3)} t_{\mathrm{c}} \geqslant 3 t_{\mathrm{pssc}}^{4}
$$

which, depending on the values of $\alpha$ and $\beta$ may or may not yield an improvement on (5.7).

\section{Discussion and numerical results}

For the plane geometry $(\lambda=0)$ with $\beta$ zero we may compare the improved lower bounds of Section 4 with the exact solution (1.4). From (1.4b) and (4.1) and (4.4) with $\beta$ zero we obtain respectively the inequalities

$$
\gamma^{-1} \geqslant\left(\alpha^{2}+\frac{\alpha}{3}\right)^{1 / 2}, \quad \gamma^{-1} \geqslant\left(\alpha^{3}+\frac{\alpha^{2}}{3}+\frac{\alpha}{15}\right)^{1 / 3}
$$


which are verified numerically in Table 1 . It is apparent from Table 1 that the new lower bounds (4.1) and (4.4) are both significant improvements on the existing pseudo steady state estimate. It is also apparent that the second improvement (4.4) for the given values of $\alpha$ is inferior to the first improvement given by (4.1). It is perhaps worth noting that the constants appearing in the sequence of bounds (6.1) are the same constants arising in the expansion (1.5) of $\alpha^{-1}$ in powers of $\gamma$, namely

$$
\alpha^{-1}=\gamma\left(1+\frac{\gamma}{3}+\frac{\gamma^{2}}{15}+\frac{\gamma^{3}}{105}+\cdots\right) .
$$

In fact we may show by induction for $\beta$ and $\lambda$ zero that for any $n \geqslant 1$ we have

$$
t^{n} \geqslant \frac{(1-R)^{2 n}}{2^{n}}\left(\alpha^{n}+\frac{\alpha^{n-1}}{3}+\cdots+\frac{2^{n-1}(n-1) !}{(2 n-1) !} \alpha\right),
$$

so that in particular for any $n \geqslant 1$ we have

$$
\gamma^{-1} \geqslant\left(\alpha^{n}+\frac{\alpha^{n-1}}{3}+\cdots+\frac{2^{n-1}(n-1) !}{(2 n-1) !} \alpha\right)^{1 / n} .
$$

However numerical results indicate that successive bounds generally decrease with increasing $n$. That is, for all but the smallest values of $\alpha, n=2$ gives the tightest lower bound. Thus even if corresponding general bounds for $\lambda=1, \lambda=2$ and $\beta$ nonzero could be established there is no reason to believe these would substantially improve the results given in Section 4.

Tables 2 and 3 give numerical values of the various upper and lower bounds for the times to complete reaction for the cylinder and sphere, respectively, for

\begin{tabular}{|c|c|c|c|c|}
\hline & \multicolumn{3}{|c|}{$\begin{array}{c}\text { Lower } \\
\text { Bounds }\end{array}$} & $\begin{array}{c}\text { Upper } \\
\text { Bound }\end{array}$ \\
\hline$\alpha$ & $(2.9)$ & $(4.7)$ & $(4.8)$ & $(2.9)$ \\
\hline 0.01 & 0.01 & 0.04 & 0.06 & 0.76 \\
\hline 0.10 & 0.08 & 0.15 & 0.14 & 0.83 \\
\hline 0.50 & 0.38 & 0.47 & 0.43 & 1.13 \\
\hline 1.00 & 0.75 & 0.85 & 0.80 & 1.50 \\
\hline 2.00 & 1.50 & 1.60 & 1.55 & 2.25 \\
\hline 5.00 & 3.75 & 3.85 & 3.80 & 4.50 \\
\hline 10.00 & 7.50 & 7.60 & 7.55 & 8.25 \\
\hline 50.00 & 37.50 & 37.60 & 37.55 & 38.25 \\
\hline 100.00 & 75.00 & 75.10 & 75.05 & 75.75 \\
\hline 500.00 & 375.00 & 375.10 & 375.05 & 375.75 \\
\hline
\end{tabular}

TABLE 2. Upper and lower bounds for time to complete reaction for $\lambda=1$ and $\beta=1$. 


\begin{tabular}{|c|c|c|c|c|}
\hline & \multicolumn{4}{|c|}{$\begin{array}{c}\text { Lower } \\
\text { Bounds }\end{array}$} \\
\hline$\alpha$ & $(2.9)$ & $(4.7)$ & $(4.8)$ & $\begin{array}{c}\text { Upper } \\
\text { Bound }\end{array}$ \\
\hline 0.01 & 0.01 & 0.03 & 0.04 & 0.51 \\
\hline 0.10 & 0.05 & 0.10 & 0.11 & 0.55 \\
\hline 0.50 & 0.25 & 0.32 & 0.30 & 0.75 \\
\hline 1.00 & 0.50 & 0.58 & 0.54 & 1.00 \\
\hline 2.00 & 1.00 & 1.08 & 1.04 & 1.50 \\
\hline 5.00 & 2.50 & 2.58 & 2.54 & 3.00 \\
\hline 10.00 & 5.00 & 5.08 & 5.03 & 5.50 \\
\hline 50.00 & 25.00 & 25.08 & 25.03 & 25.50 \\
\hline 100.00 & 50.00 & 50.08 & 50.03 & 50.50 \\
\hline 500.00 & 250.00 & 250.08 & 250.03 & 250.50 \\
\hline
\end{tabular}

TABLE 3. Upper and lower bounds for time to complete reaction for $\lambda=2$ and $\beta=1$.

various values of $\alpha$. It is apparent from these tables that for all but the smallest values of $\alpha$ the first improvement given by (4.7) is superior, as a lower bound, to the second improvement given by (4.8). This is also the case for the plane geometry with $\beta$ nonzero.

Finally we remark that the procedure described here is not effective for problems with nonlinear diffusivity. For this case we refer the reader to the bounds obtained in [2] for the nonlinear diffusivity problem with no radiation $(\beta=0)$.

\section{References}

[1] G. B. Davis and J. M. Hill, "A moving boundary problem for the sphere", IMA J. Appl. Math. 29 (1982), 99-111.

[2] J. N. Dewynne and J. M. Hill, "On an integral formulation for moving boundary problems", Quart. Appl. Math. 42 (1984), 443-455.

[3] D. Glasser and J. Kern, "Bounds and approximate solutions to linear problems with non-linear boundary conditions", AIChE.J. 24 (1978), 161-170.

[4] J. M. Hill, "On the pseudo steady state approximation for moving boundary diffusion problems", Chem. Engrg. Sci. 39 (1984), 187-190.

[5] J. R. Ockendon and W. R. Hodgkins (eds.), Moving boundary problems in heatflow and diffusion (Clarendon Press, Oxford, 1975).

[6] L. I. Rubinstein, The Stefan problem, Translations of Mathematical Monographs 27 (Amer. Math. Soc., Providence, R. I., 1971).

[7] D. G. Wilson, A. D. Solomon and P. T. Boggs (eds.), Moving boundary problems (Academic Press, New York, 1978). 